\title{
Synthesis of rapidly surface eroding polyorthoesters and polyacetals using thiol-ene click chemistry
}

\author{
Gordon Herwig ${ }^{\dagger, *}$ and Andrew P. Dove ${ }^{\dagger, *}$ \\ ${ }^{\dagger}$ School of Chemistry, The University of Birmingham, Edgbaston, Birmingham, UK, B15 2TT \\ ${ }^{\ddagger}$ School of Chemistry, University of Warwick, Gibbet Hill, Coventry, CV4 7AL
}

\section{Supporting Information}

\begin{abstract}
Materials
Acetone (Fisher), Aliquat 336 (Sigma-Aldrich), basic aluminium oxide (Sigma-Aldrich), 2-bromo-1,1-dimethoxyethane (Acros Organics), cis-2-butene-1,4-diol (Sigma-Aldrich), diethyl ether (Sigma-Aldrich), 3,6-dioxa-1,8-octane-dithiol (Sigma-Aldrich), ethanol (Sigma-Aldrich), ethanethiol (Sigma-Aldrich), hexane-1,6-dithiol (Sigma-Aldrich), hydrochloric acid (Fisher), iodoacetamide (Sigma-Aldrich), Irgacure 819 (IGM Resins Ltd.), potassium carbonate (anhydrous, Sigma-Aldrich), potassium tert-butoxide (Sigma-Aldrich), 1,3-propanediol (Sigma-Aldrich), terephthalic acid (Sigma-Aldrich), tetrahydrofuran (Sigma-Aldrich), tributylphosphine (Sigma-Aldrich), triethylamine (Sigma-Aldrich), preparation for Dulbecco's PBS (Sigma-Aldrich) and p-toluenesulfonic acid (Sigma) were used as received from the supplier. $\mathrm{CDCl}_{3}$ (Sigma-Aldrich) was stored over anhydrous potassium carbonate and plug filtered through basic aluminium oxide immediately before use. Furthermore, all glassware used for handling and storage of OE containing compounds was silanized by treating the inner surface with Sigmacote (Sigma-Aldrich) for five minutes, followed by rinsing with water, acetone, diethyl ether and subsequent drying under compressed air for one hour.
\end{abstract}

\section{Methods}

A Formlabs Form Cure or an NK Optik Otoflash was used for curing of polymers. Nuclear Magnetic Resonance (NMR) spectra were acquired using a Bruker Avance III $400 \mathrm{MHz}$, processed by MestReNova software using the residual solvent peak as reference $\left(\mathrm{CDCl}_{3},{ }^{1} \mathrm{H}: \delta=7.26 \mathrm{ppm},{ }^{13} \mathrm{C}: \delta=77.16\right.$ ppm). Mass spectra were obtained via a Bruker maXis plus. Elemental analysis was conducted on a CE Instruments EA1110 Elemental Analyzer. Size Exclusion Chromatograms were recorded on an Agilent Infinity II with RI detection on an Agilent PLgel MIXED-C, $7.5 \times 300 \mathrm{~mm}, 5 \mu \mathrm{m}$ column in THF with $2 \% \mathrm{NEt}_{3}$ and $0.01 \% \mathrm{BHT}$ at a flow rate of $1 \mathrm{~mL} \cdot \mathrm{min}^{-1}$ at $50{ }^{\circ} \mathrm{C}$ compared to EasiVial Polystyrene Standard. Agilent GPC/SEC software V.A.02.01 (2015) was used for data analysis. An Agilent Cary 630 FTIR Spectrometer with Agilent Resolutions Pro software V.5.0 was used for IR spectroscopy. DSC and TGA thermograms were collected on a Mettler-Toledo STARe DSC3+ system and a TA Instruments TGA Q500, respectively. Oscillatory rheometry was performed on an Anton Parr MCR-302 with PP25 plates, Omnicure S1500 UV source and RheoCompass ${ }^{\mathrm{Tm}}$ V1.20.496 software at a frequency of $10 \mathrm{~Hz}$, amplitude of $1 \%$ and gap width of $0.2 \mathrm{~mm}$. AFM images were recorded on a JPK Nanowizard 4 in QI mode using Nanosensors PPP-NCHAuD tips and Nanowizard Control Software V.6.1.117. SEM images were obtained on a Philips XL30 ESEM-FEG Environmental SEM using Phoenix Software V.3.3 on gold-coated samples.

Bis(3-mercaptopropyl) succinate was synthesized according to established procedure within the group. Briefly, 3-mercaptopropanol (7.30 g, 0.079 mol) and succinic acid $(4.40 \mathrm{~g}, 0.037 \mathrm{~mol})$ are dissolved in $120 \mathrm{~mL}$ of a 1:1 mixture of benzene and toluene with a catalytic amount of sulphuric acid. After stirring at $120^{\circ} \mathrm{C}$ overnight and collection of the distilled water, the solution was left to cool to room temperature, followed by vacuum transfer of the solvent to yield crude product. The oil was re-dissolved in chloroform, extracted with sat. $\mathrm{NaHCO}_{3}$ solution $(2 \times 200 \mathrm{~mL})$ and dried over $\mathrm{MgSO} 4$. Purification on a silica gel column $(3: 2$ hexane $/ E t O A c)$ and subsequent distillation $\left(0.2 \mathrm{mbar}, 220^{\circ} \mathrm{C}\right)$ yielded clear colorless oil ( $7.8 \mathrm{~g}, 79 \%$ yield). $\mathrm{R}_{\mathrm{f}}$ $(3: 2 \mathrm{Hex} / \mathrm{EtOAc})=0.4,{ }^{1} \mathrm{H} \mathrm{NMR}\left(400 \mathrm{MHz} ; 298 \mathrm{~K} ; \mathrm{CDCl}_{3} ; \mathrm{ppm}\right): \delta 4.21\left(\mathrm{t},{ }^{3} \mathrm{JH}=6.2 \mathrm{~Hz}, 4 \mathrm{H}\right), 2.62(\mathrm{~s}, 4 \mathrm{H}), 2.58\left(\mathrm{q},{ }^{3} J_{\mathrm{HH}}=6.6 \mathrm{~Hz}, 4 \mathrm{H}\right), 1.40(\mathrm{t}$, $\left.{ }^{3} J_{\mathrm{HH}}=8.1 \mathrm{~Hz}, 2 \mathrm{H}\right) ;{ }^{13} \mathrm{C} \mathrm{NMR}\left(400 \mathrm{MHz} ; 298 \mathrm{~K} ; \mathrm{CDCl}_{3} ; \mathrm{ppm}\right): \delta 172.31,62.94,32.92,29.22,21.20 ; \mathrm{MS}$ (ESI-QTOF +ve): $(\mathrm{M}+\mathrm{Na})^{+} m / z$ calculated for $\mathrm{C}_{10} \mathrm{H}_{18} \mathrm{O}_{4} \mathrm{~S}_{2} \mathrm{Na}^{+}$: 289.1, found: 289.0; Anal. Calcd for $\mathrm{C}_{10} \mathrm{H}_{18} \mathrm{O}_{4} \mathrm{~S}_{2}$ : C 45.09; $\mathrm{H} 6.81 \%$. Found: C 59.70; $\mathrm{H} 4.41 \%$.

Synthesis of 2-(bromomethyl)-1,3-dioxe-5-pene (BrCKA, 1). The procedure follows a method described by Plikk et al. ${ }^{1}$ Cis-2-butene-1,4-diol (33.30 g, $378 \mathrm{mmol})$ and 2-bromo-1,1-dimethoxyethane $(63.89 \mathrm{~g}, 378 \mathrm{mmol})$ were weighed into a $100 \mathrm{~mL}$ round bottom flask, followed by p-toluenesulfonic acid $(0.10 \mathrm{~g}, 0.1 \mathrm{wt} \%)$. A stirrer bar, vigreux condenser and distillation bridge was added and the mixture incrementally heated to $130^{\circ} \mathrm{C}$ under stirring, until no more methanol was distilled over. After cooling to room temperature and gradually reducing the pressure, a residual methanol fraction was discarded and subsequently the pure product distilled at $38-42{ }^{\circ} \mathrm{C}$ and $0.05 \mathrm{mbar}(62.1 \mathrm{~g}, 85 \%)$. Characterized as reported previously. ${ }^{1} \mathrm{H}$ NMR 
$\left(400 \mathrm{MHz} ; 298 \mathrm{~K} ; \mathrm{CDCl}_{3} ; \mathrm{ppm}\right): \delta 5.70\left(\mathrm{t},{ }^{3} J_{\mathrm{HH}}=1.5 \mathrm{~Hz}, 2 \mathrm{H}\right), 4.97\left(\mathrm{t},{ }^{3} J_{\mathrm{HH}}=5.4 \mathrm{~Hz}, 1 \mathrm{H}\right), 4.44\left(\mathrm{dm},{ }^{3} J_{\mathrm{HH}}=14.7 \mathrm{~Hz}, 2 \mathrm{H}\right), 4.20\left(\mathrm{dm},{ }^{3} J_{\mathrm{HH}}=14.7 \mathrm{~Hz}\right.$, $2 \mathrm{H}), 3.39\left(\mathrm{~d},{ }^{3} \mathrm{~J}_{\mathrm{HH}}=5.4 \mathrm{~Hz}, 2 \mathrm{H}\right) ;{ }^{13} \mathrm{C} \mathrm{NMR}\left(400 \mathrm{MHz} ; 298 \mathrm{~K} ; \mathrm{CDCl}_{3} ; \mathrm{ppm}\right): \delta 128.81,106.92,63.25,24.99$.

Synthesis of 2-methylene-1,3-dioxe-5-pene (CKA, 2). Similarly to Plikk et al'.s method, ${ }^{1} \mathrm{BrCKA}(1)$ (40.00 g, $\left.207 \mathrm{mmol}\right)$ and Aliquat 336 (1.62 g, $\left.4 \mathrm{mmol}\right)$ were weighed into a $500 \mathrm{~mL} \mathrm{RBF}$, dissolved in $350 \mathrm{~mL}$ THF and cooled under stirring to $0^{\circ} \mathrm{C}$. Potassium tert-butoxide $(46.50 \mathrm{~g}, 414 \mathrm{mmol}) \mathrm{was}$ added in small portions within one hour and the orange to yellow suspension left to stir for another two hours at $0{ }^{\circ} \mathrm{C}$. THF was removed in vacuo $(20 \mathrm{mbar}$, $18^{\circ} \mathrm{C}$ ) and the residual slurry dissolved in $200 \mathrm{~mL}$ diethyl ether and $200 \mathrm{~mL}$ distilled water, followed by separation of the layers and extraction of the aqueous phase with a further $100 \mathrm{~mL}$ diethyl ether. The combined organic layers were washed with $0.01 \mathrm{M}$ potassium carbonate solution ( $300 \mathrm{~mL})$ for three times. The organic layer was concentrated in vacuo at $18^{\circ} \mathrm{C}$, followed by vacuum transfer of the residual oil at room temperature and $0.05 \mathrm{mbar}$ to yield crude product. Repeat of the washing procedure, followed by drying over potassium carbonate and concentration at $30^{\circ} \mathrm{C}$ and $20 \mathrm{mbar}$ yielded $\begin{array}{llll}\text { pure } & \text { product } & (12.50 & \mathrm{g}, \quad 5 \%)\end{array}$ ${ }^{1} \mathrm{H} \mathrm{NMR}\left(400 \mathrm{MHz} ; 298 \mathrm{~K} ; \mathrm{CDCl}_{3} ; \mathrm{ppm}\right): \delta 5.72\left(\mathrm{t},{ }^{3} \mathrm{JHH}=1.7 \mathrm{~Hz}, 2 \mathrm{H}\right), 4.43\left(\mathrm{~d},{ }^{3} \mathrm{JHH}_{\mathrm{HH}}=1.7 \mathrm{~Hz}, 4 \mathrm{H}\right), 3.57(\mathrm{~s}, 2 \mathrm{H}) ;{ }^{13} \mathrm{C} \mathrm{NMR}\left(400 \mathrm{MHz} ; 298 \mathrm{~K} ; \mathrm{CDCl}_{3}\right.$; $\mathrm{ppm}): \delta 164.11,127.83,68.77,68.21$.

General procedure of the orthoester (OE) synthesis (3).CKA (2) and diol were weighed into a silanized vial in a molar ratio of 1.05 to 0.5 . Terephthalic acid ( 1 mol eq.) was added and the mixture stirred overnight. The crude product was dissolved in diethyl ether and washed four times with equivalent volumes of $0.01 \mathrm{M}$ potassium carbonate solution, followed by separation and drying of the organic layer over potassium carbonate. Plug filtration through minimal amounts of basic alumina and evaporation yielded pure OE product of fruity odour (3). Additional distillation in vacuo in a silanized setup before use was performed to remove any degradation products (around ${ }^{1} \mathrm{H}$ NMR $\delta 2.00 \mathrm{ppm}$ ).

Synthesis of 2-ethoxy-2-methyl-1,3-dioxe-5-pene (EtOE, 3a).

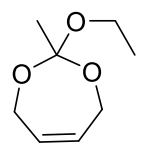

The reaction of CKA (2) $(2.00 \mathrm{~g}, 17.84 \mathrm{mmol})$, ethanol $(0.82 \mathrm{~g}, 8.92 \mathrm{mmol})$ and terephthalic acid $(0.30 \mathrm{~g}, 0.18 \mathrm{mmol})$ yielded clear colourless oil (2.20 g, 78\%; distillation at $\left.0.05 \mathrm{mbar}, 60{ }^{\circ} \mathrm{C}\right) .{ }^{1} \mathrm{H} \mathrm{NMR}\left(400 \mathrm{MHz} ; 298 \mathrm{~K} ; \mathrm{CDCl}_{3} ; \mathrm{ppm}\right): \delta 5.65\left(\mathrm{t},{ }^{3} \mathrm{JHH}_{\mathrm{HH}} 1.7 \mathrm{~Hz}, 2 \mathrm{H},-\mathrm{CHCH}_{2} \mathrm{CO}_{3-}\right), 4.44\left(\mathrm{dm},{ }^{2} \mathrm{JHH}_{\mathrm{HH}}=-\right.$ $\left.16.4 \mathrm{~Hz}, 2 \mathrm{H},-\mathrm{CHCH}_{2} \mathrm{CO}_{3}-\right), 4.13\left(\mathrm{dm}^{2}{ }^{2} \mathrm{HHH}_{\mathrm{HH}}=-16.4 \mathrm{~Hz}, 2 \mathrm{H},-\mathrm{CHCH}_{2} \mathrm{CO}_{3}-\right), 3.58\left(\mathrm{q},{ }^{3} J_{\mathrm{HH}}=7.0 \mathrm{~Hz}, 2 \mathrm{H}, \mathrm{CH}_{3} \mathrm{CH}_{2} \mathrm{CO}_{3-}\right), 1.53\left(\mathrm{~s}, 3 \mathrm{H}, \mathrm{CH}_{3} \mathrm{CO}_{3}-\right), 1.24$ $\left(\mathrm{t},{ }^{3} \mathrm{JHH}_{\mathrm{HH}}=7.0 \mathrm{~Hz}, 3 \mathrm{H}, \mathrm{CH}_{3} \mathrm{CH}_{2} \mathrm{CO}_{3^{-}}\right) ;{ }^{13} \mathrm{C} \mathrm{NMR}\left(400 \mathrm{MHz} ; 298 \mathrm{~K} ; \mathrm{CDCl}_{3} ; \mathrm{ppm}\right): \delta 128.99\left(-\mathrm{CHCH}_{2} \mathrm{CO}_{3^{-}}\right), 116.08\left(-\mathrm{CO}_{3^{-}}\right), 61.47\left(-\mathrm{CHCH}_{2} \mathrm{CO}_{3-}\right)$, $58.89\left(\mathrm{CH}_{3} \mathrm{CH}_{2} \mathrm{CO}_{3-}^{-}\right), 19.15\left(\mathrm{CH}_{3} \mathrm{CO}_{3-}^{-}\right), 15.49\left(\mathrm{CH}_{3} \mathrm{CH}_{2} \mathrm{CO}_{3-}\right)$; MS (ESI-QTOF +ve): $(\mathrm{M}+\mathrm{Na})^{+} m / z$ calculated for $\mathrm{C}_{8} \mathrm{H}_{14} \mathrm{O}_{3} \mathrm{Na}^{+}: 181.0835$, found: 181.0836; Anal. Calcd for $\mathrm{C}_{8} \mathrm{H}_{14} \mathrm{O}_{3}$ : C 60.7; H 8.9\%. Found: C 60.2; H 8.9; IR (neat; $\left.298 \mathrm{~K}_{\text {; cm}}^{-1}\right): 2980-2870(-\mathrm{C}-\mathrm{H}), 1380\left(-\mathrm{CH} 2^{-}\right), 1160-1040(\mathrm{C}-$ O), $900\left(-\mathrm{CH}_{2}-\mathrm{CH}_{2}-\right), 800(\mathrm{C}=\mathrm{C}), 640(=\mathrm{C}-\mathrm{H})$

Synthesis of 1,3-bis(2-methyl-2-oxyl-1,3-dioxe-5-pene)-propane (OE3, 3b).<smiles>CC1(OCCCOC2(C)OCC=CCO2)OCC=CCO1</smiles>

CKA (2) (4.00 g, $35.68 \mathrm{mmol})$, 1,3-propanediol (1.36 g, $17.84 \mathrm{mmol})$ and terephthalic acid $(0.60 \mathrm{~g}, 0.36 \mathrm{mmol})$ were reacted to yield clear colourless oil (4.48 g, 84\%; distillation at $\left.0.02 \mathrm{mbar}, 150{ }^{\circ} \mathrm{C}\right) .{ }^{1} \mathrm{H} \mathrm{NMR}\left(400 \mathrm{MHz} ; 298 \mathrm{~K} ; \mathrm{CDCl}_{3} ; \mathrm{ppm}\right): \delta 5.66\left(\mathrm{t},{ }^{3} \mathrm{HHH}_{1}=1.7 \mathrm{~Hz}, 4 \mathrm{H},-\mathrm{CHCH}_{2} \mathrm{CO}_{3}-\right), 4.43(\mathrm{dm}$, $\left.4 \mathrm{H},{ }^{2} J_{\mathrm{HH}}=-15.5 \mathrm{~Hz},-\mathrm{CHCH}_{2} \mathrm{CO}_{3}-\right), 4.15\left(\mathrm{dm}, 4 \mathrm{H},{ }^{2} J_{\mathrm{HH}}=-15.5 \mathrm{~Hz},-\mathrm{CHCH}_{2} \mathrm{CO}_{3}-\right), 3.65\left(\mathrm{t}, 4 \mathrm{H},{ }^{3} J_{\mathrm{HH}}=6.3 \mathrm{~Hz},-\mathrm{OCH}_{2} \mathrm{CH}_{2}-\right), 1.93\left(q \mathrm{qi}, 2 \mathrm{H},{ }^{3} J_{\mathrm{HH}}=6.3 \mathrm{~Hz}\right.$, $\left.-\mathrm{OCH}_{2} \mathrm{CH}_{2-}\right), 1.54\left(\mathrm{~s}, 6 \mathrm{H},-\mathrm{CO}_{3} \mathrm{CH}_{3}\right) ;{ }^{13} \mathrm{CNMR}\left(400 \mathrm{MHz} ; 298 \mathrm{~K} ; \mathrm{CDCl}_{3} ; \mathrm{ppm}\right): \delta 128.99\left(-\mathrm{CHCH}_{2} \mathrm{CO}_{3}-\right), 116.20\left(-\mathrm{CO}_{3} \mathrm{CH}_{3}\right), 61.57(-$ $\left.\left.\mathrm{CHCH}_{2} \mathrm{CO}_{3}\right)^{-}\right), 60.42\left(-\mathrm{CO}_{3} \mathrm{CH}_{2} \mathrm{CH}_{2-}\right), 30.37\left(-\mathrm{CO}_{3} \mathrm{CH}_{2} \mathrm{CH}_{2-}\right), 19.18\left(-\mathrm{CO}_{3} \mathrm{CH}_{3}\right)$; MS (ESI-QTOF +ve): $(\mathrm{M}+\mathrm{Na})^{+} \mathrm{m} / z$ calculated for $\mathrm{C}_{15} \mathrm{H}_{24} \mathrm{O}_{6} \mathrm{Na}^{+}: 323.1465$, found: 323.1468; Anal. Calcd for $\mathrm{C}_{15} \mathrm{H}_{24} \mathrm{O}_{6}: \mathrm{C} 60.0 ; \mathrm{H} 8.05 \%$. Found: C 59.9; H 8.0; IR (neat; $\left.298 \mathrm{~K} ; \mathrm{cm}^{-1}\right): 2950-2860(-\mathrm{C}-$ $\mathrm{H}), 1380\left(-\mathrm{CH}_{2}-\right), 1160-1040(\mathrm{C}-\mathrm{O}), 900\left(-\mathrm{CH}_{2}-\mathrm{CH}_{2}-\right), 800(\mathrm{C}=\mathrm{C}), 640(=\mathrm{C}-\mathrm{H})$

Synthesis of cis-1,3-bis(2-methyl-2-oxyl-1,3-dioxe-5-pene)-but-2-ene (OE4ene, 3c).

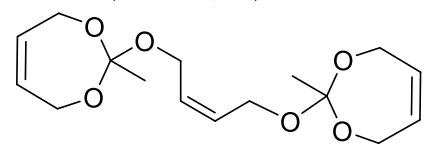

The reaction of CKA (2) (2.00 g, $17.84 \mathrm{mmol})$, cis-2-butene-1,4-diol (0.78 g, $8.92 \mathrm{mmol})$ and terephthalic acid $(0.30 \mathrm{~g}, 0.18 \mathrm{mmol})$ yielded white crystals (2.25 g, 81\%; recrystallisation from diethyl ether). $\mathrm{Mp} . \approx 25^{\circ} \mathrm{C} ;{ }^{1} \mathrm{H} \mathrm{NMR}\left(400 \mathrm{MHz} ; 298 \mathrm{~K} ; \mathrm{CDCl}_{3} ; \mathrm{ppm}\right): \delta 5.71\left(\mathrm{tm},{ }^{3} \mathrm{JHH}_{\mathrm{HH}}=3.6 \mathrm{~Hz}, 2 \mathrm{H},-\right.$ $\mathrm{CHCH}_{2} \mathrm{CO}_{3^{-}}$(linker)), $5.56\left(\mathrm{t},{ }^{3} \mathrm{JHH}_{\mathrm{HH}}=1.7 \mathrm{~Hz}, 4 \mathrm{H},-\mathrm{CHCH}_{2} \mathrm{CO}_{3-}\right), 4.43\left(\mathrm{dm}, 4 \mathrm{H},{ }^{2} J_{\mathrm{HH}}=-14.2 \mathrm{~Hz},-\mathrm{CHCH}_{2} \mathrm{CO}_{3^{-}}\right), 4.15\left(\mathrm{~d}, 4 \mathrm{H},{ }^{3} J_{\mathrm{HH}}=3.6 \mathrm{~Hz},-\right.$ $\mathrm{CHCH}_{2} \mathrm{CO}_{3}-($ linker $\left.)\right), 4.13\left(\mathrm{dm}, 4 \mathrm{H},{ }^{2} J_{\mathrm{HH}}=-14.2 \mathrm{~Hz},-\mathrm{CHCH}_{2} \mathrm{CO}_{3}-\right), 1.54\left(\mathrm{~s}, 6 \mathrm{H},-\mathrm{CO}_{3} \mathrm{CH}_{3}\right) ;{ }^{13} \mathrm{C} \mathrm{NMR}\left(400 \mathrm{MHz} ; 298 \mathrm{~K} ; \mathrm{CDCl}_{3} ; \mathrm{ppm}\right): \delta 128.94$ $\left(-\mathrm{CHCH}_{2} \mathrm{CO}_{3}-\right), 128.64\left(-\mathrm{CHCH}_{2} \mathrm{CO}_{3-}\right.$ (linker $\left.)\right), 116.35\left(-\mathrm{CO}_{3} \mathrm{CH}_{3}\right), 61.70\left(-\mathrm{CHCH}_{2} \mathrm{CO}_{3}-\right), 59.72\left(-\mathrm{CHCH}_{2} \mathrm{CO}_{3-}(\right.$ linker $\left.)\right), 30.37\left(-\mathrm{CO}_{3} \mathrm{CH}_{2} \mathrm{CH}_{2-}\right.$ ), $19.40\left(-\mathrm{CO}_{3} \mathrm{CH}_{3}\right)$; MS (ESI-QTOF +ve): $(\mathrm{M}+\mathrm{Na})^{+} \mathrm{m} / z$ calculated for $\mathrm{C}_{16} \mathrm{H}_{24} \mathrm{O}_{6} \mathrm{Na}^{+}: 335.1465$, found: 335.1468; Anal. Calcd for $\mathrm{C}_{16} \mathrm{H}_{24} \mathrm{O}_{6}: \mathrm{C}$ 61.5; H 7.7\%. Found: C 61.5; H 7.8; IR (neat; $\left.298 \mathrm{~K} ; \mathrm{cm}^{-1}\right): 2940-2870(-\mathrm{C}-\mathrm{H}), 1380\left(-\mathrm{CH}_{2}-\right), 1160-1020(\mathrm{C}-\mathrm{O}), 900\left(-\mathrm{CH}_{2}-\mathrm{CH}_{2}-\right), 800(\mathrm{C}=\mathrm{C}), 640$ $(=\mathrm{C}-\mathrm{H})$ 
General procedure of the acetal (AT) synthesis (4). CKA (2) and thiol were weighed into a silanized vial under a nitrogen blanket in a molar ratio of 1.05 to 0.5 . Irgacure $819(0.3 \mathrm{wt} \%)$ was added and the mixture stirred until clear, followed by irradiation with UV light for $5 \mathrm{~h}$ at $60^{\circ} \mathrm{C}$ in a Formlabs Form Cure. Vacuum distillation yielded the pure AT product.

Synthesis of 2-((ethylthio)methyl)-1,3-dioxe-5-pene (EtAT, 4a).<smiles>CCSCC1OCC=CCO1</smiles>

CKA (2) $(2.10 \mathrm{~g}, 18.73 \mathrm{mmol})$, ethanethiol $(1.10 \mathrm{~g}, 8.92 \mathrm{mmol})$ and Irgacure $819(0.01 \mathrm{~g})$ were reacted to yield clear colourless oil (3.02 g, $97 \%$; distillation at $\left.0.02 \mathrm{mbar}, 60^{\circ} \mathrm{C}\right) .{ }^{1} \mathrm{H} \mathrm{NMR}\left(400 \mathrm{MHz} ; 298 \mathrm{~K} ; \mathrm{CDCl}_{3} ; \mathrm{ppm}\right): \delta 5.71\left(\mathrm{t},{ }^{3} \mathrm{HHH}_{\mathrm{HH}}=1.8 \mathrm{~Hz}, 2 \mathrm{H},-\mathrm{CHCH}_{2} \mathrm{CHO}_{2}\right), 4.91\left(\mathrm{t}, 1 \mathrm{H},{ }^{3} J_{\mathrm{HH}}=5.5 \mathrm{~Hz}\right.$, $\left.-\mathrm{CHO}_{2} \mathrm{CH}_{2} \mathrm{~S}-\right), 4.42\left(\mathrm{dm}, 2 \mathrm{H},{ }^{2} J_{\mathrm{HH}}=-15.2 \mathrm{~Hz},-\mathrm{CHCH}_{2} \mathrm{CHO}_{2}-\right), 4.19\left(\mathrm{dm}, 2 \mathrm{H}^{2}{ }^{2} J_{\mathrm{HH}}=-15.2 \mathrm{~Hz},-\mathrm{CHCH}_{2} \mathrm{CHO}_{2}-\right), 2.75\left(\mathrm{~d}, 2 \mathrm{H},{ }^{3} J_{\mathrm{HH}}=5.5 \mathrm{~Hz}^{-}\right.$ $\left.\mathrm{CHO}_{2} \mathrm{CH}_{2} \mathrm{~S}-\right), 2.62\left(\mathrm{~d}, 2 \mathrm{H},{ }^{3} J_{\mathrm{HH}}=7.4 \mathrm{~Hz},-\mathrm{SCH}_{2} \mathrm{CH}_{3}\right), 1.25\left(\mathrm{~s}, 3 \mathrm{H},{ }^{3} J_{\mathrm{HH}}=7.4 \mathrm{~Hz},-\mathrm{SCH}_{2} \mathrm{CH}_{3}\right) ;{ }^{13} \mathrm{C} \mathrm{NMR}\left(400 \mathrm{MHz} ; 298 \mathrm{~K} ; \mathrm{CDCl}_{3} ; \mathrm{ppm}^{2}: \delta 129.58\right.$ $\left(-\mathrm{CHCH}_{2} \mathrm{CHO}_{2}-\right), 104.35\left(-\mathrm{CHO}_{2} \mathrm{CH}_{2} \mathrm{~S}-\right), 65.59\left(-\mathrm{CHCH}_{2} \mathrm{CHO}_{2}-\right), 34.66\left(-\mathrm{CHO}_{2} \mathrm{CH}_{2} \mathrm{~S}-\right), 26.73\left(-\mathrm{SCH}_{2} \mathrm{CH}_{3}\right), 14.81\left(-\mathrm{SCH}_{2} \mathrm{CH}\right)$; $\mathrm{MS}(\mathrm{ESI}-\mathrm{QTOF}$ $+v e): \mathrm{M}^{+} m / z$ calculated for $\mathrm{C}_{8} \mathrm{H}_{14} \mathrm{O}_{2} \mathrm{~S}^{+}: 174.0715$, found: 174.0716; Anal. Calcd for $\mathrm{C}_{8} \mathrm{H}_{14} \mathrm{O}_{2} \mathrm{~S}$ : C 55.1; $\mathrm{H}$ 8.1; $\mathrm{S}$ 18.4\%. Found: C 55.55; $\mathrm{H}$ 8.1; $\mathrm{S} 18.0$; IR (neat; $\left.298 \mathrm{~K} ; \mathrm{cm}^{-1}\right): 2950-2850(-\mathrm{C}-\mathrm{H}), 1440-1350\left(-\mathrm{CH}_{2}-\right), 1120-980(\mathrm{C}-\mathrm{O}), 910(=\mathrm{C}-\mathrm{H}), 740(=\mathrm{C}-\mathrm{H})$

Synthesis of 1,6-bis(2-methylthioyl-1,3-dioxe-5-pene)-hexane (AT6, 4b).

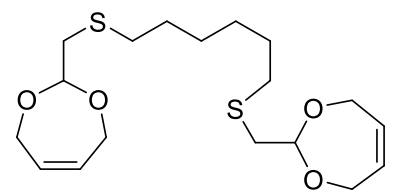

The reaction of CKA (2) (2.10 g, $18.73 \mathrm{mmol})$, 1,6-hexanedithiol (1.34 g, $8.92 \mathrm{mmol})$ and Irgacure 819 (0.01 g) yielded clear colourless oil (3.21 g, $96 \%$; distillation at $\left.0.02 \mathrm{mbar}, 150{ }^{\circ} \mathrm{C}\right) .{ }^{1} \mathrm{H} \mathrm{NMR}\left(400 \mathrm{MHz} ; 298 \mathrm{~K}_{;} \mathrm{CDCl}_{3} ; \mathrm{ppm}\right): \delta 5.71\left(\mathrm{t},{ }^{3} J_{\mathrm{HH}}=1.8 \mathrm{~Hz}, 4 \mathrm{H},-\mathrm{CHCH}_{2} \mathrm{CHO}_{2-}\right), 4.90\left(\mathrm{t}, 2 \mathrm{H},{ }^{3} J_{\mathrm{HH}}=\right.$ $\left.5.5 \mathrm{~Hz},-\mathrm{CHO}_{2} \mathrm{CH}_{2} \mathrm{~S}-\right), 4.42\left(\mathrm{dm}, 4 \mathrm{H},{ }^{2} \mathrm{JHH}_{\mathrm{HH}}=-15.5 \mathrm{~Hz},-\mathrm{CHCH}_{2} \mathrm{CHO}_{2}-\right), 4.19\left(\mathrm{dm}, 4 \mathrm{H},{ }^{2} \mathrm{JHH}_{\mathrm{HH}}=-15.5 \mathrm{~Hz},-\mathrm{CHCH}_{2} \mathrm{CHO}_{2}-\right), 2.73\left(\mathrm{~d}, 4 \mathrm{H}^{3}{ }^{3} \mathrm{JHH}_{\mathrm{HH}}=5.5 \mathrm{~Hz}\right.$, $\left.-\mathrm{CHO}_{2} \mathrm{CH}_{2} \mathrm{~S}-\right), 2.59\left(\mathrm{~d}, 4 \mathrm{H},{ }^{3} \mathrm{JHH}_{\mathrm{HH}}=7.5 \mathrm{~Hz},-\mathrm{SCH}_{2} \mathrm{CH}_{2} \mathrm{CH}_{2}-\right), 1.59\left(\mathrm{~m}, 4 \mathrm{H},-\mathrm{SCH}_{2} \mathrm{CH}_{2} \mathrm{CH}_{2}-\right), 1.39\left(\mathrm{~m}, 4 \mathrm{H},-\mathrm{SCH}_{2} \mathrm{CH}_{2} \mathrm{CH}_{2}-\right)^{13} \mathrm{C} \mathrm{NMR}^{13}\left(400 \mathrm{MHz}^{-}\right.$ $298 \mathrm{~K} ; \mathrm{CDCl}_{3}$; ppm): $\delta 129.60\left(-\mathrm{CHCH}_{2} \mathrm{CHO}_{2-}\right), 104.38\left(-\mathrm{CHO}_{2} \mathrm{CH}_{2} \mathrm{~S}-\right), 65.62\left(-\mathrm{CHCH}_{2} \mathrm{CHO}_{2}-\right), 35.13\left(-\mathrm{CHO}_{2} \mathrm{CH}_{2} \mathrm{~S}_{-}\right), 32.80\left(-\mathrm{SCH}_{2} \mathrm{CH}_{2} \mathrm{CH}_{2-}\right)$, $29.54\left(-\mathrm{SCH}_{2} \mathrm{CH}_{2} \mathrm{CH}_{2}-\right), 28.47\left(-\mathrm{SCH}_{2} \mathrm{CH}_{2} \mathrm{CH}_{2}-\right)$; $\mathrm{MS}$ (ESI-QTOF +ve): $(\mathrm{M}+\mathrm{Na})^{+} \mathrm{m} / z$ calculated for $\mathrm{C}_{18} \mathrm{H}_{30} \mathrm{O}_{4} \mathrm{~S}_{2} \mathrm{Na}^{+}: 397.1478$, found: 397.1307; Anal. Calcd for $\mathrm{C}_{18} \mathrm{H}_{30} \mathrm{O}_{4} \mathrm{~S}_{2}$ : C 57.7; H 8.1; S 17.1\%. Found: C 57.7; H 8.9; S 16.9; IR (neat; $298 \mathrm{~K}$ cm $\left.\mathrm{cm}^{-1}\right): 2920-2850$ (-C-H), 1440-1350 (-CH $\left.2^{-}\right)$, $1120-990(\mathrm{C}-\mathrm{O}), 910(=\mathrm{C}-\mathrm{H}), 750\left(-\mathrm{CH}_{2}-\right)$

General procedure of the thiol-ene synthesis to form small molecule and chain thio-ethers $(T E)(\mathbf{5 , 6})$. The respective OE or AT $(3,4)$ and thiol were weighed into a silanized vial under a nitrogen blanket in a molar ratio of 1.0 to 0.5 . Irgacure 819 ( $0.3 \mathrm{wt} \%$ for small molecules, $1.0 \mathrm{wt} \%$ for polymers $)$ was added and the mixture stirred under ambient light for one day until colourless. After vigorous stirring, the viscous preparations were subjected to $24 \mathrm{~h}$ of $\mathrm{UV}$ light at $60^{\circ} \mathrm{C}$ in a Formlabs Form Cure. Small molecules were finally distilled in vacuo to yield pure product, whereas polymeric products were generally used as is for further analysis.

Synthesis of 2-ethoxy-5-(hexylthio)-2-methyl-1,3-dioxepane (EtOE-TE, 5a).

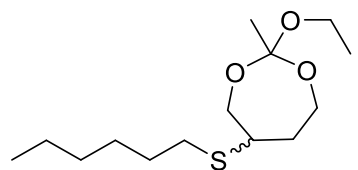

The reaction of EtOE $(3 \boldsymbol{a})(2.00 \mathrm{~g}, 12.64 \mathrm{mmol})$, 1-hexanethiol $(1.49 \mathrm{~g}, 12.64 \mathrm{mmol})$ and Irgacure $819(0.01 \mathrm{~g})$ yielded clear oily product (3.30 g, 94\%; distillation at $\left.0.02 \mathrm{mbar}, 150{ }^{\circ} \mathrm{C}\right) .{ }^{1} \mathrm{H}$ NMR $\left(400 \mathrm{MHz} ; 298 \mathrm{~K} ; \mathrm{CDCl}_{3} ; \mathrm{ppm}\right): \delta 3.96-3.42(\mathrm{~m}, 4 \mathrm{H}), 3.48 / 3.46\left(\mathrm{q},{ }^{3} \mathrm{JH}=7.1 \mathrm{~Hz}, 2 \mathrm{H}\right), 2.80 / 2.72$ $(\mathrm{m}, 1 \mathrm{H}), 2.46(\mathrm{~m}, 2 \mathrm{H}), 1.95-1.55(\mathrm{~m}, 2 \mathrm{H}), 1.55-1.45(\mathrm{~m}, 3 \mathrm{H}), 1.37 / 1.35(\mathrm{~s}, 3 \mathrm{H}), 1.34-1.18(\mathrm{~m}, 3 \mathrm{H}), 1.16 / 1.14\left(\mathrm{t},{ }^{3} \mathrm{HH}=7.1 \mathrm{~Hz}, 3 \mathrm{H}\right), 0.82(\mathrm{~s}, 3 \mathrm{H})$; ${ }^{13} \mathrm{C}$ NMR (400 MHz; $\left.298 \mathrm{~K} ; \mathrm{CDCl}_{3} ; \mathrm{ppm}\right): \delta 115.72 / 115.64,66.60 / 64.99,61.81 / 59.77,58.70 / 58.62,43.98 / 43.21,36.61 / 35.69,31.51 / 31.48$, 31.23/31.04, 30.15/30.09, 28.71/28.68, 22.63, 20.71/20.64, 15.49, 14.13; MS (ESI-QTOF +ve): $(\mathrm{M}+\mathrm{Na})^{+} \mathrm{m} / z$ calculated for $\mathrm{C}_{14} \mathrm{H}_{28} \mathrm{NO}_{3} \mathrm{SNa}^{+}$: 299.1651, found: 299.1657; Anal. Calcd for $\mathrm{C}_{14} \mathrm{H}_{28} \mathrm{O}_{3}$ S: C 60.8; H 10.2; S 11.6\%. Found: C 60.8; H 10.3; S 11.5; IR (neat; 298 K; $\left.\mathrm{cm}^{-1}\right): 2930-2860$ (C-H), $1380\left(-\mathrm{CH}_{2}-\right), 1160-1030$ (C-O)

Synthesis of 2-((ethylthio)methyl)-5-(hexylthio)-1,3-dioxepane (EtAT-TE, 6a). 


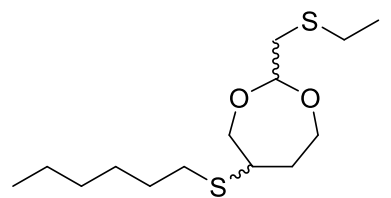

EtAT (4a) (2.00 g, $11.48 \mathrm{mmol})$, 1-hexanethiol $(1.36 \mathrm{~g}, 11.48 \mathrm{mmol})$ and Irgacure $819(0.01 \mathrm{~g})$ were reacted to yield clear oily product (3.22 g, $95 \%$; distillation at $\left.0.02 \mathrm{mbar}, 150{ }^{\circ} \mathrm{C}\right) .{ }^{1} \mathrm{H} \mathrm{NMR}\left(400 \mathrm{MHz} ; 298 \mathrm{~K} ; \mathrm{CDCl}_{3} ; \mathrm{ppm}\right): \delta 4.90-4.84(\mathrm{~m}, 1 \mathrm{H}), 4.12-3.51(\mathrm{~m}, 4 \mathrm{H}), 2.94-2.78(\mathrm{~m}, 1 \mathrm{H}), 2.69-2.58$ $(\mathrm{m}, 2 \mathrm{H}), 2.11-1.95(\mathrm{~m}, 1 \mathrm{H}), 2.58-2.48(\mathrm{~m}, 2 \mathrm{H}), 2.11-1.95(\mathrm{~m}, 1 \mathrm{H}), 1.76-1.62(\mathrm{~m}, 1 \mathrm{H}), 1.61-1.50(\mathrm{~m}, 2 \mathrm{H}), 1.42-1.23(\mathrm{~m}, 6 \mathrm{H}), 1.26\left(\mathrm{qm},{ }^{3} J_{\mathrm{HH}}=7.2\right.$ $\mathrm{Hz}, 2 \mathrm{H}), 0.88\left(\mathrm{tm},{ }^{3} J_{\mathrm{HH}}=7.2 \mathrm{~Hz}, 3 \mathrm{H}\right) ;{ }^{13} \mathrm{C} \mathrm{NMR}\left(400 \mathrm{MHz} ; 298 \mathrm{~K} ; \mathrm{CDCl}_{3} ; \mathrm{ppm}\right): \delta 102.74 / 102.36,70.91 / 66.53,65.53 / 62.11,44.18 / 43.84,36.29$, 35.01/34.68, 31.54/31.50, 31.24/31.18, 30.09/30.06, 28.69, 26.73, 22.63, 14.81, 14.13.; MS (ESI-QTOF +ve): $(\mathrm{M}+\mathrm{K})^{+} \mathrm{m} / z$ calculated for $\mathrm{C}_{14} \mathrm{H}_{28} \mathrm{O}_{2} \mathrm{~S}_{2} \mathrm{~K}^{+}$: 331.1168, found: 331.1170; Anal. Calcd for $\mathrm{C}_{14} \mathrm{H}_{28} \mathrm{O}_{2} \mathrm{~S}_{2}$ : C 57.5; H 9.65; S 21.9\%. Found: C 59.3; H 9.2; S 21.5; IR (neat; $298 \mathrm{~K}$; cm 1): 2920-2850 (-C-H), 1450-1340 (- $\left.\mathrm{CH}_{2}-\right), 1120-990(\mathrm{C}-\mathrm{O}), 740\left(-\mathrm{CH}_{2}-\right)$

Synthesis of poly\{[1,3-bis(2-methyl-2-oxyl-5-yl-1,3-dioxepane)-O-propane]-alt-(1,6-thioyl-hexane)\}(OE3-2TE6, 5b).

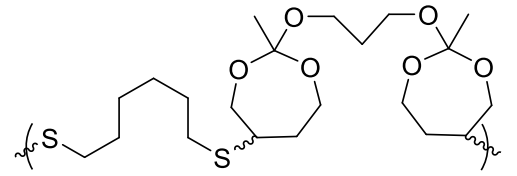

The reaction of OE3 $(3 \boldsymbol{b})(1.00 \mathrm{~g}, 3.33 \mathrm{mmol}), 1,6$-hexanedithiol $(0.50 \mathrm{~g}, 3.33 \mathrm{mmol})$ and Irgacure $819(0.015 \mathrm{~g})$ yielded clear viscous product. ${ }^{1} \mathrm{H}$ NMR $\left(400 \mathrm{MHz} ; 298 \mathrm{~K} ; \mathrm{CDCl}_{3} ; \mathrm{ppm}\right): \delta 4.02-3.77(\mathrm{~m}, 4 \mathrm{H}), 3.69-3.50(\mathrm{~m}, 8 \mathrm{H}), 2.91-2.73(\mathrm{~m}, 2 \mathrm{H}), 2.56-2.47(\mathrm{~m}, 4 \mathrm{H}), 2.02-1.93(\mathrm{~m}, 2 \mathrm{H}), 1.93-$ $1.82(\mathrm{~m}, 2 \mathrm{H}), 1.78-1.50(\mathrm{~m}, 6 \mathrm{H}), 1.45-1.41(\mathrm{~ms}, 6 \mathrm{H}), 1.41-1.34(\mathrm{~m}, 4 \mathrm{H}) ;{ }^{13} \mathrm{C} \mathrm{NMR}\left(400 \mathrm{MHz} ; 298 \mathrm{~K} ; \mathrm{CDCl}_{3} ; \mathrm{ppm}\right): \delta 115.88,66.71 / 65.07$, $61.91 / 60.32,60.26 / 59.86,44.06 / 43.27,36.63 / 35.72,31.16 / 31.00,30.30,30.03 / 30.00,28.63,20.69$; IR (neat; 298 K; $\left.\mathrm{cm}^{-1}\right): 2920-2850(-\mathrm{C}-\mathrm{H}), 1370$ $\left(-\mathrm{CH}_{2}-\right), 1150-1020(\mathrm{C}-\mathrm{O}), 900\left(-\mathrm{CH}_{2}-\mathrm{CH}_{2}-\right)$; GPC (THF): $M_{\mathrm{n}}=4400 \mathrm{~g} \cdot \mathrm{mol}^{-1}\left(\bigoplus_{\mathrm{M}}=2.6\right)$

Synthesis of poly\{[1,3-bis(2-methyl-2-oxyl-5-yl-1,3-dioxepane)-O-propane $]$-alt-(3,6-dioxa-1,8-dithioyl-octane)\} (OE3-2TEeg, 5c).

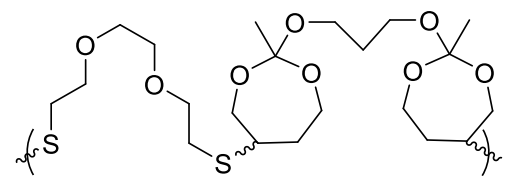

OE3 (3b) (1.00 g, $3.33 \mathrm{mmol})$ was reacted with 3,6-dioxa-1,8-octane-dithiol ( $0.61 \mathrm{~g}, 3.33 \mathrm{mmol})$ and Irgacure $819(0.016 \mathrm{~g})$ to yield clear viscous product. ${ }^{1} \mathrm{H}$ NMR $(400 \mathrm{MHz} ; 298 \mathrm{~K} ; \mathrm{CDCl} 3 ; \mathrm{ppm}): \delta 4.16\left(\mathrm{t},{ }^{3} \mathrm{HH}_{\mathrm{HH}}=6.3 \mathrm{~Hz}, 4 \mathrm{H}\right), 3.99-3.79(\mathrm{~m}, 4 \mathrm{H}), 3.67-3.50(\mathrm{~m}, 8 \mathrm{H}), 2.91-2.74(\mathrm{~m}, 2 \mathrm{H}), 2.63(\mathrm{~s}$, $4 \mathrm{H}), 2.61-2.53(\mathrm{~m}, 4 \mathrm{H}), 2.02-1.93(\mathrm{~m}, 2 \mathrm{H}), 1.93-1.83(\mathrm{~m}, 6 \mathrm{H}), 1.75-1.57(\mathrm{~m}, 2 \mathrm{H}), 1.48-1.37(\mathrm{~ms}, 6 \mathrm{H}) ;{ }^{13} \mathrm{C} \mathrm{NMR}(400 \mathrm{MHz} ; 298 \mathrm{~K} ; \mathrm{CDCl} 3$; ppm): $\delta 115.87 / 115.78,71.36 / 71.18,70.47,66.58 / 65.05,61.70 / 59.76,60.32 / 60.28,44.40 / 43.64,36.65 / 35.75,30.62 / 30.51,30.29,20.72 ;$ IR (neat; 298 K; $\left.\mathrm{cm}^{-1}\right): 2940-2870(-\mathrm{C}-\mathrm{H}), 1380\left(-\mathrm{CH}_{2}-\right), 1150-960(\mathrm{C}-\mathrm{O}), 900\left(-\mathrm{CH}_{2}-\mathrm{CH}_{2}-\right) ; \mathrm{GPC}(\mathrm{THF}): M_{\mathrm{n}}=4600 \mathrm{~g} \cdot \mathrm{mol}^{-1}\left(\bigoplus_{\mathrm{M}}=2.3\right)$

Synthesis of poly\{[1,3-bis(2-methyl-2-oxyl-5-yl-1,3-dioxepane)-O-propane]-alt-[bis(3-thioylpropyl) succinate]\} (OE3-2TEsucc, 5d).<smiles>CC1(C)CCOC(C)(OCCCOC2(C)OCC[C@H](SCCCOC(=O)CCC(=O)OCCCSC(C)(C)C)CO2)OC1</smiles>

OE3 $(3 \boldsymbol{b})(1.00 \mathrm{~g}, 3.33 \mathrm{mmol})$, bis(3-mercaptopropyl) succinate $(8.87 \mathrm{~g}, 3.33 \mathrm{mmol})$ and Irgacure $819(0.019 \mathrm{~g})$ were combined in the reaction to yielded clear viscous product. ${ }^{1} \mathrm{H}$ NMR $(400 \mathrm{MHz} ; 298 \mathrm{~K}$; CDCl3; ppm): $\delta 4.00-3.75(\mathrm{~m}, 4 \mathrm{H}), 3.75-3.60(\mathrm{~m}, 8 \mathrm{H}), 3.60-3.59(\mathrm{~s}, 4 \mathrm{H}), 3.59-3.48(\mathrm{~m}$, $4 \mathrm{H}), 2.97-2.80(\mathrm{~m}, 2 \mathrm{H}), 2.79-2.65(\mathrm{~m}, 4 \mathrm{H}), 2.03-1.93(\mathrm{~m}, 2 \mathrm{H}), 1.93-1.80(\mathrm{~m}, 2 \mathrm{H}), 1.74-1.55(\mathrm{~m}, 2 \mathrm{H}), 1.46-1.39(\mathrm{~ms}, 6 \mathrm{H}) ;{ }^{13} \mathrm{C} \mathrm{NMR}(400 \mathrm{MHz} ; 298$ $\left.\mathrm{K} ; \mathrm{CDCl}_{3} ; \mathrm{ppm}\right): \delta 172.33,115.88,66.52 / 64.96,63.39 / 63.35,61.75 / 59.79,60.32 / 60.26,44.12 / 43.39,36.52 / 35.63,30.29,29.19,29.13,27.59 / 27.48$, 20.66; IR (neat; $\left.298 \mathrm{~K} ; \mathrm{cm}^{-1}\right): 2940-2880(-\mathrm{C}-\mathrm{H}), 1730(\mathrm{C}=\mathrm{O}) ; 1380\left(-\mathrm{CH}_{2}-\right), 1150-960(\mathrm{C}-\mathrm{O}), 900\left(-\mathrm{CH}_{2}-\mathrm{CH}_{2}-\right)$; GPC $(\mathrm{THF}): M_{\mathrm{n}}=5400 \mathrm{~g} \cdot \mathrm{mol}^{-1}$ $\left(\bigoplus_{\mathrm{M}}=2.3\right)$

Synthesis of poly\{[1,3-bis(2-methyl-2-oxyl-5-yl-1,3-dioxepane)-O-propane $]$-alt-(dithioylthreitol)\} (OE3-2TEdtt, Se).<smiles>CC1CCOC(C)(OCCCOC2(C)OCC[C@H](SCC[C@H](O)[C@@H](O)CCC(C)(C)C)CO2)CC1</smiles>

The reaction of OE3 $(3 \boldsymbol{b})(1.00 \mathrm{~g}, 3.33 \mathrm{mmol})$, dithiothreitol $(0.51 \mathrm{~g}, 3.33 \mathrm{mmol})$ and Irgacure $819(0.015 \mathrm{~g})$ yielded an oily liquid. ${ }^{1} \mathrm{H}$ NMR spectroscopy and SEC chromatography suggest only low conversion. GPC (THF): $M_{\mathrm{n}}=500 \mathrm{~g} \cdot \mathrm{mol}^{-1}\left(\bigoplus_{\mathrm{M}}=2.5\right)$

Synthesis of poly\{[1,6-bis(2-methylthioyl-5-yl-1,3-dioxepane)-S-hexane $]$-alt-(1,6-thioyl-hexane) $\}$ (AT6-2TE6, 6b). 


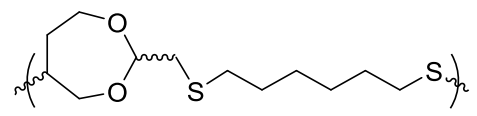

The reaction of AT6 (4b) (1.00 g, $3.20 \mathrm{mmol})$, 1,6-hexanedithiol $(0.40 \mathrm{~g}, 2.67 \mathrm{mmol})$ and Irgacure $819(0.014 \mathrm{~g})$ yielded clear viscous product. ${ }^{1} \mathrm{H} \mathrm{NMR}\left(400 \mathrm{MHz} ; 298 \mathrm{~K} ; \mathrm{CDCl}_{3} ; \mathrm{ppm}\right): \delta 4.91-4.78(2 \mathrm{t}, 1 \mathrm{H}), 4.13-3.95(\mathrm{~m}, 1 \mathrm{H}), 3.91-3.70(\mathrm{~m}, 2 \mathrm{H}), 3.66-3.50(\mathrm{~m}, 1 \mathrm{H}), 2.93-2.76(\mathrm{~m}, 1 \mathrm{H}), 2.67-$ $2.60(\mathrm{~m}, 2 \mathrm{H}), 2.60-2.47(\mathrm{~m}, 4 \mathrm{H}), 2.10-1.95(\mathrm{~m}, 1 \mathrm{H}), 1.73-1.63(\mathrm{~m}, 1 \mathrm{H}), 1.63-1.48(\mathrm{~ms}, 4 \mathrm{H}), 1.44-1.33(\mathrm{~ms}, 4 \mathrm{H}) ;{ }^{13} \mathrm{C} \mathrm{NMR}\left(400 \mathrm{MHz} ; 298 \mathrm{~K} ; \mathrm{CDCl}_{3}\right.$; ppm): $\delta 102.36 / 102.36,70.88 / 66.59,65.52 / 62.15,44.26 / 43.92,36.30,35.49 / 35.22,32.83 / 32.81,31.15 / 31.13,31.09 / 29.94,29.56 / 29.54$, 29.53/28.49; IR (neat; $\left.298 \mathrm{~K} ; \mathrm{cm}^{-1}\right): 2910-2850(-\mathrm{C}-\mathrm{H}), 1450-1340\left(-\mathrm{CH}_{2}-\right), 1120-990(\mathrm{C}-\mathrm{O}), 750\left(-\mathrm{CH}_{2-}\right) ; \mathrm{GPC}(\mathrm{THF}): M_{\mathrm{n}}=5900 \mathrm{~g} \cdot \mathrm{mol}^{-1}\left(\bigoplus_{\mathrm{M}}=\right.$ $3.8)$

Reduction and end-capping of polymer chains. Disulfide linkages were reduced to thiol groups and protected from oxidation following an adapted procedure in the style of commercially available ReadyPrep ${ }^{\text {tm }}$ Reduction-Alkylation Kits. The sample was completely dissolved in THF with triethylamine (2\%). Under a nitrogen blanket tributylphosphine $(200 \mathrm{~mol} \mathrm{eq}$.) was added and the sealed vial shaken vigorously $(300 \mathrm{rpm})$ for $4 \mathrm{~h}$, followed by addition of a solution of iodoacetamide ( $300 \mathrm{~mol} \mathrm{eq.)} \mathrm{in} \mathrm{THF} \mathrm{and} \mathrm{further} \mathrm{shaking} \mathrm{for} 4 \mathrm{~h}$. Water $(2 \mathrm{vol} \mathrm{eq}$.) was added and the suspension centrifuged to form a clear organic phase. Separation, washing with water and drying in the SEC solvent over potassium carbonate yielded the final chain polymer product.

Synthesis of net-poly\{[1,3-bis(2-methyl-2-oxyl-5-yl-1,3-dioxepane)-2-yl-O-butane]-co-(1,6-thioyl-hexane)\} (OE4ene-2TE6, 7).

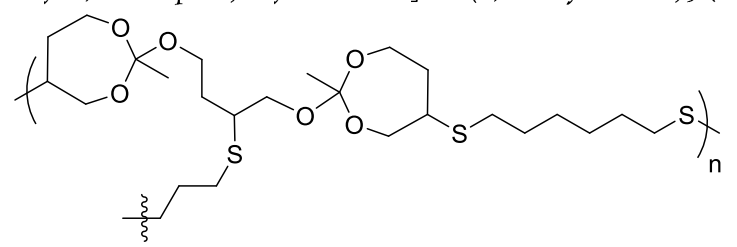

A mixture of OE4ene (3c) $(2.00 \mathrm{~g}, 6.40 \mathrm{mmol})$, 1,6-hexanedithiol (1.44 g, $9.6 \mathrm{mmol})$ and Irgacure $819(0.034 \mathrm{~g}, 1 \mathrm{wt} \%)$ was stirred in a silanized brown vial under a nitrogen blanket until the solution cleared. $0.3 \mathrm{~mL}$ of the solution was then each deposited into a PP mold ( $2 \mathrm{~mL}$ vial cap with lowered rim) to form a smooth top surface. Subsequent curing in an Otoflash G171 flash box for five times 1000 flashes with intermittent cooling, followed by extraction from the molds and a further five times 1000 flashes, yielded the polymer material in clear cylindrical shapes. $T_{\mathrm{g}}=10.2^{\circ} \mathrm{C}\left(\mathrm{DSC}, 10 \mathrm{~K} \cdot \mathrm{min}^{-1}\right.$, heat); IR (neat; $\left.298 \mathrm{~K} ; \mathrm{cm}^{-1}\right): 2910-2840(-\mathrm{C}-\mathrm{H}), 1370\left(-\mathrm{CH}_{2}-\right), 1150-1000$ (C-O), $900\left(-\mathrm{CH}_{2}-\mathrm{CH}_{2-}\right)$ 


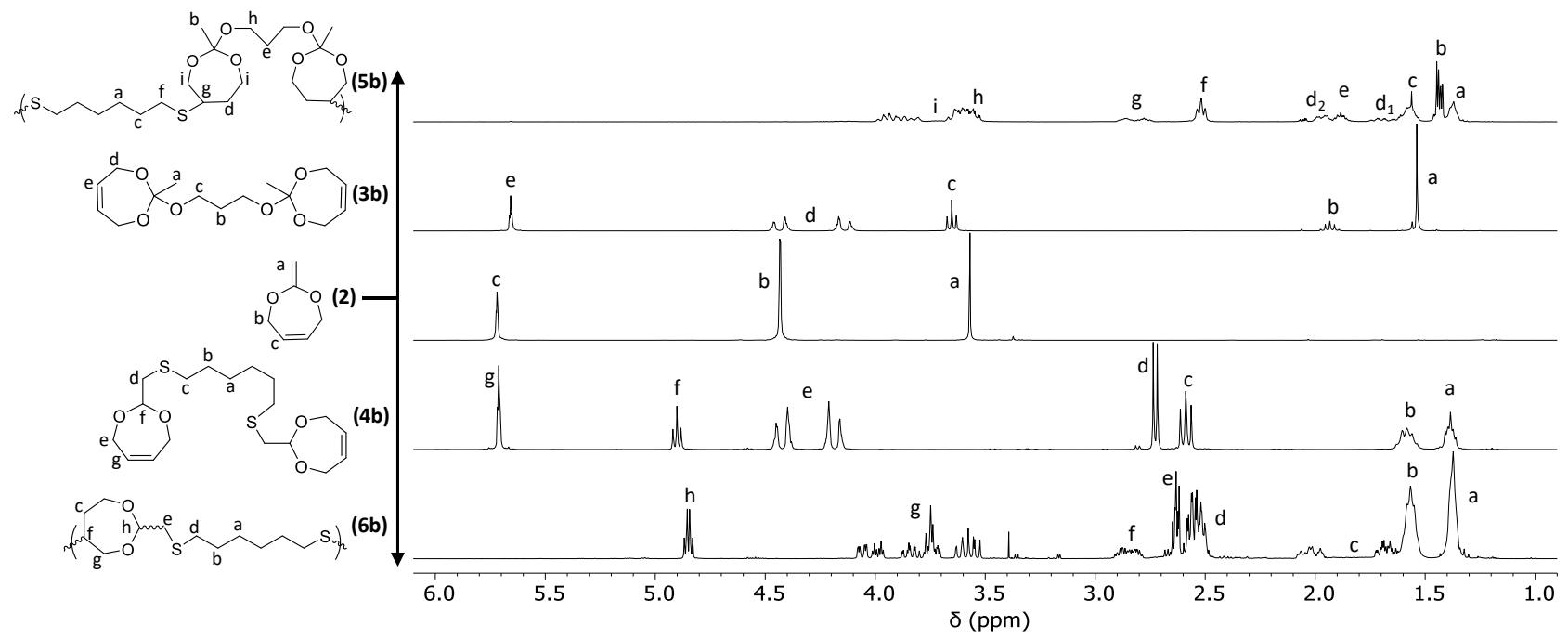

Figure S1. ${ }^{1} \mathrm{H}$ NMR spectra of dimeric OE (3b) and AT (4b), as well as the respective chain polymers (5b, 6b) $\left(\mathrm{CDCl}_{3}, 400 \mathrm{MHz}, 298 \mathrm{~K}\right)$.

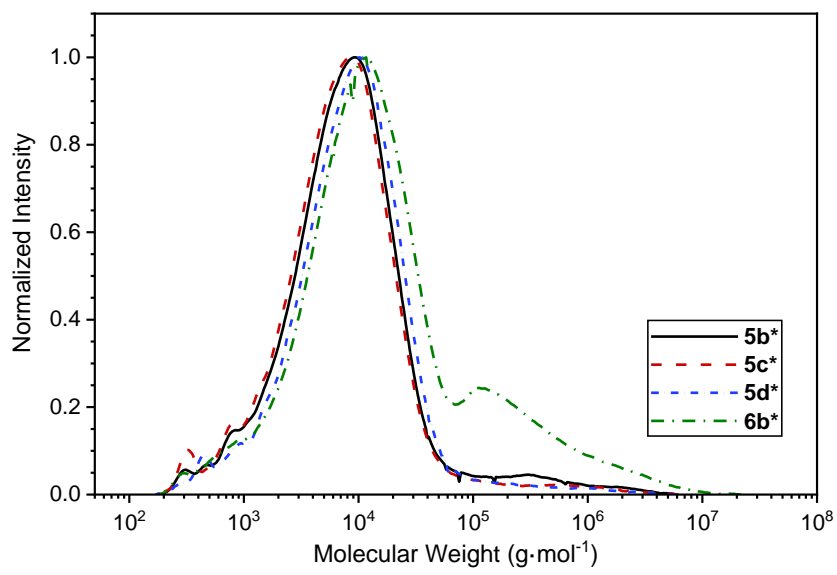

Figure S2. Size Exclusion Chromatograms of different POETE (5b-d) and PATTE (6b) chain polymers (crude, molecular weight determined in THF with $2 \% \mathrm{NEt}_{3}$ against polystyrene standards).

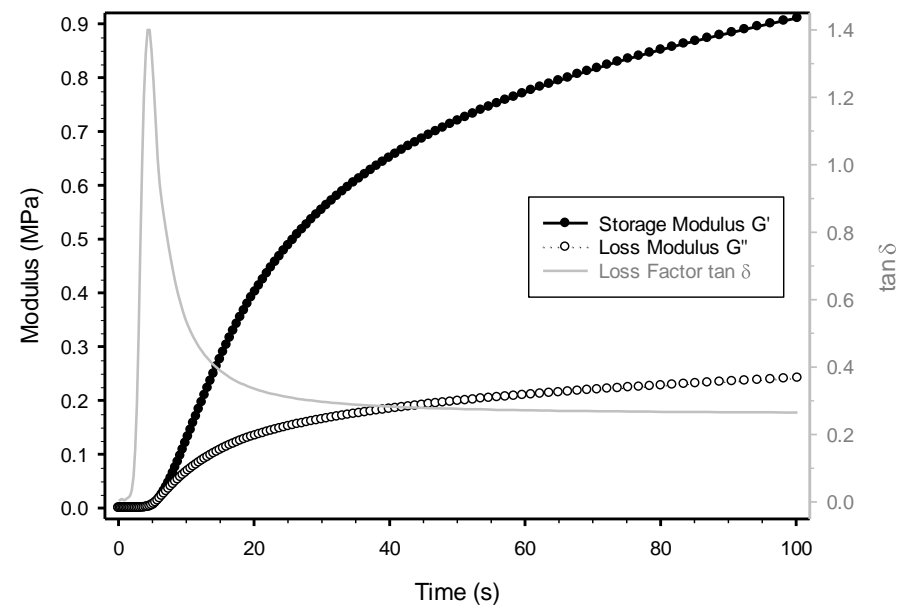

Figure S3. Progression of oscillatory storage and loss modulus during the polymerisation of P(OE4ene-2TE6) (7) (frequency $10 \mathrm{~Hz}$, amplitude $1 \%$, gap width $0.2 \mathrm{~mm}$ ). 

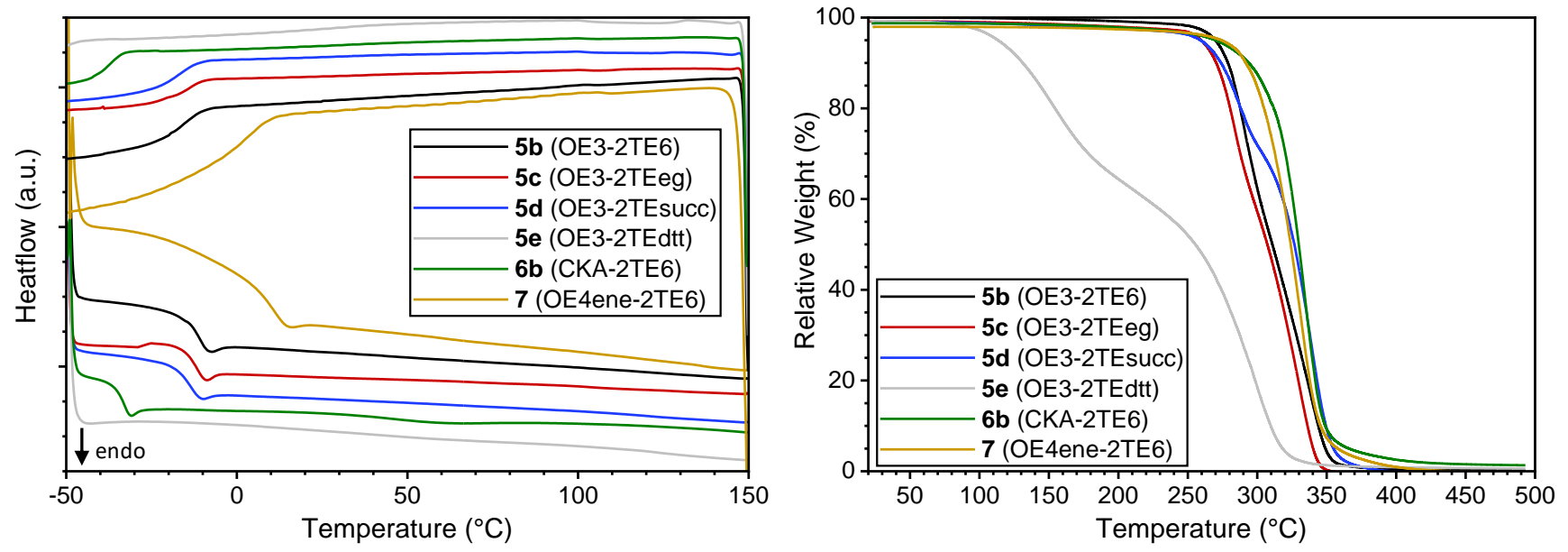

Figure S4. DSC (left, $3^{\text {rd }}$ cycle) and TGA (right) thermograms of all polymeric compounds $(\mathbf{5 b}-\mathbf{7}) .\left(10 \mathrm{~K} \cdot \mathrm{min}^{-1}\right)$.
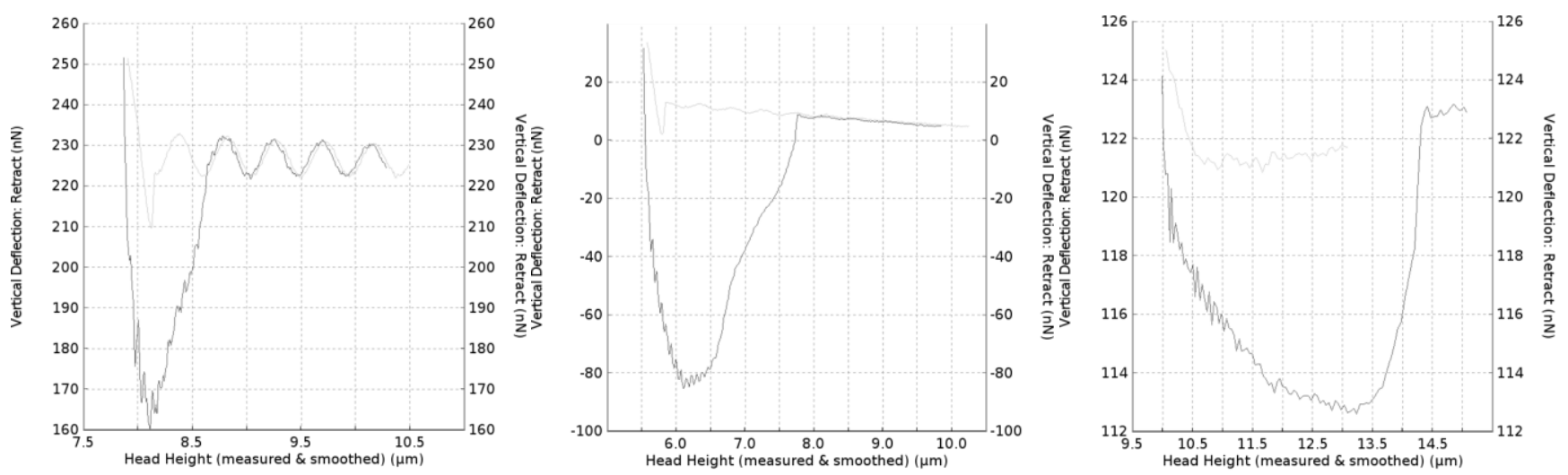

Figure S5. Exemplary AFM Force curves on degraded samples of $\mathrm{P}(\mathrm{OE} 4 \mathrm{ene}-2 \mathrm{TE} 6)$ (7) at roughly $50 \mathrm{wt} \%$.

From left to right: crude; PBS; $0.1 \mathrm{M} \mathrm{HCl}(25 \mathrm{nN}$, retraction maxed at $15 \mu \mathrm{m})$. Grey: approach; black: retract.
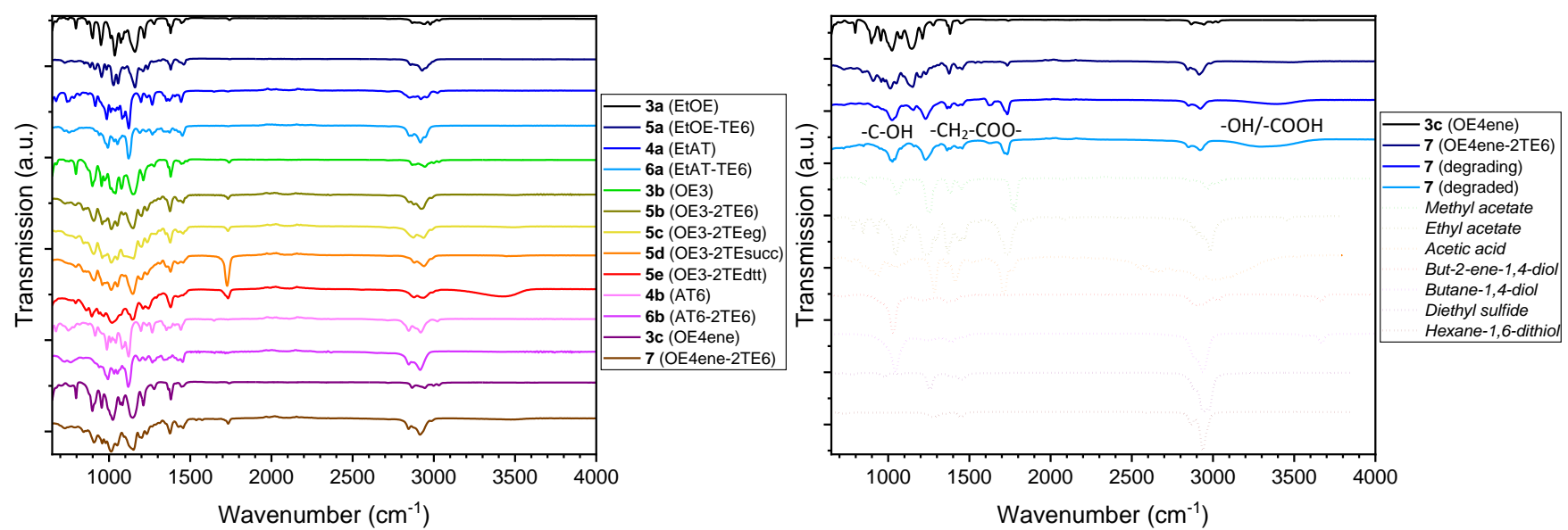

Figure S6. FT-IR spectra of all new compounds (3a-7, left) and degradation products of P(OE4ene-2TE6) (7, right) on air (neat). Spectra of reference substances (dashed, right) obtained from the NIST (National Institute of Standards and Technology) database. 

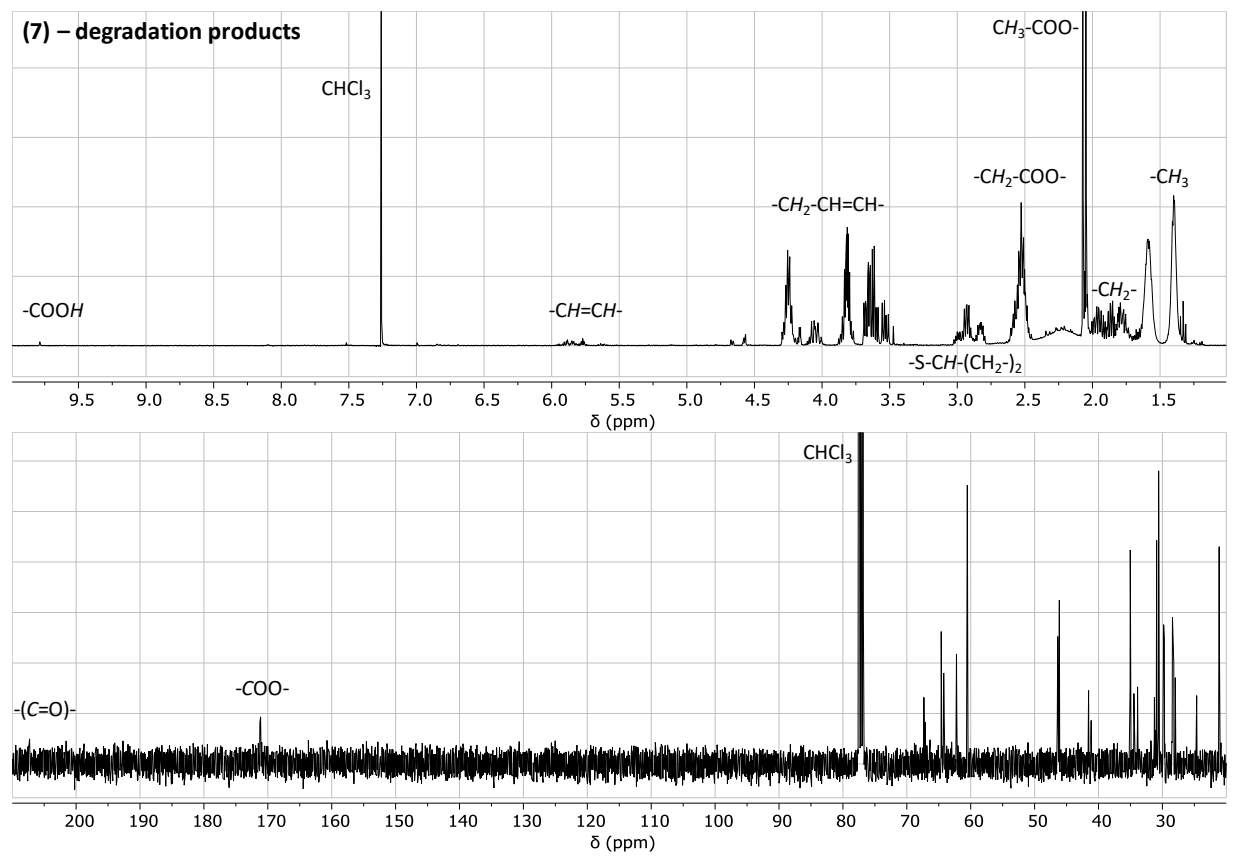

Figure S7. ${ }^{1} \mathrm{H}$ (top) and ${ }^{13} \mathrm{C}$ (bottom) NMR spectra of degradation products of the $\mathrm{P}(\mathrm{OE} 4 \mathrm{ene}-2 \mathrm{TE} 6)(7)$ on air $\left(\mathrm{CDCl}_{3}, 400 \mathrm{MHz}, 298 \mathrm{~K}\right)$. 


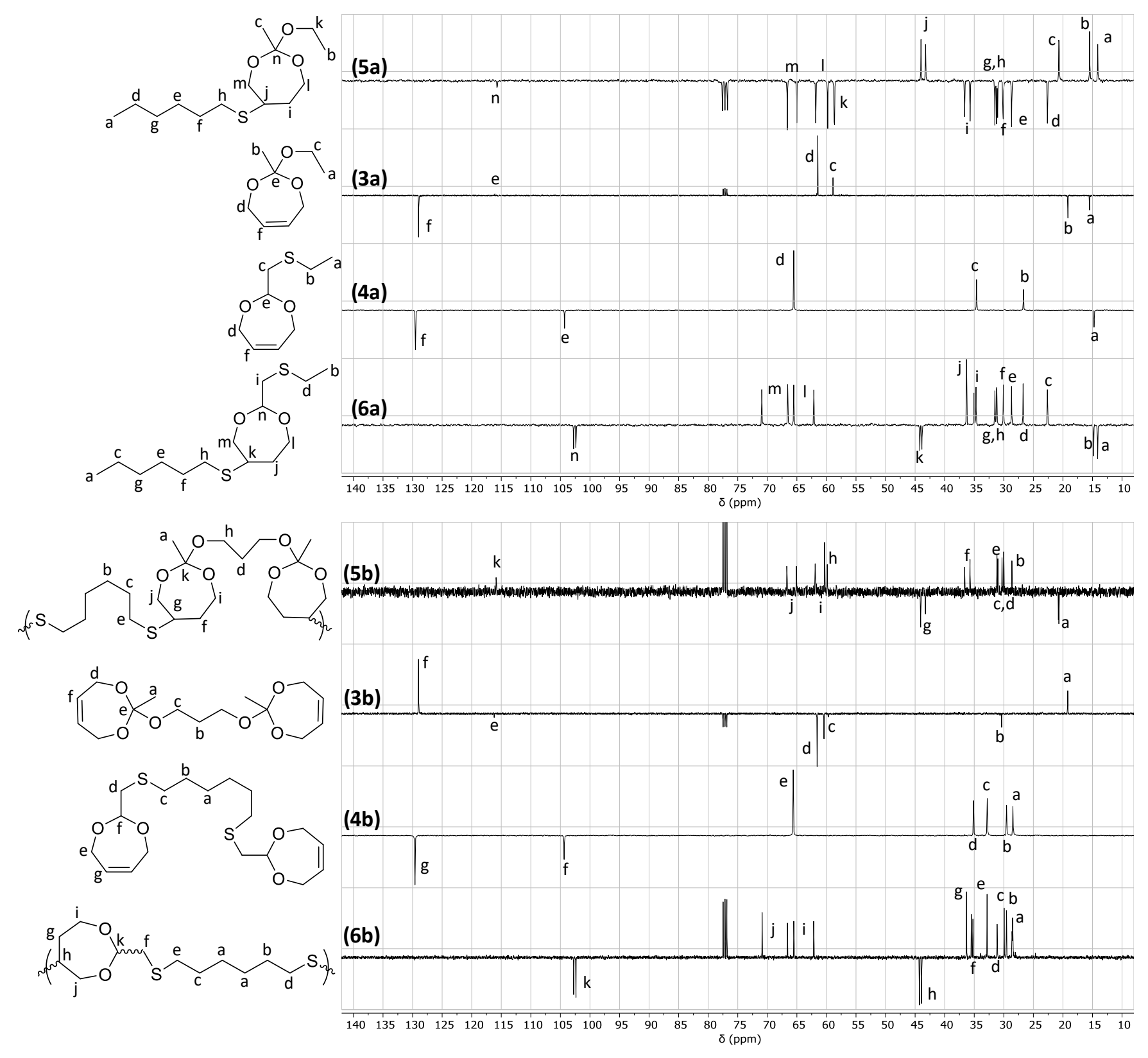

Figure S8. ${ }^{13} \mathrm{C}$ NMR spectra of selected OEs (3a, 3b), ATs (4a, 4b) and respective TEs $(\mathbf{5 a}, \mathbf{5 b}, \mathbf{6 a}, \mathbf{6 b})\left(\mathrm{CDCl}_{3}, 300 \mathrm{MHz}, 298 \mathrm{~K}\right)$. Compounds and arrangement analogous to Figures 1 and $\mathrm{S} 1$. 


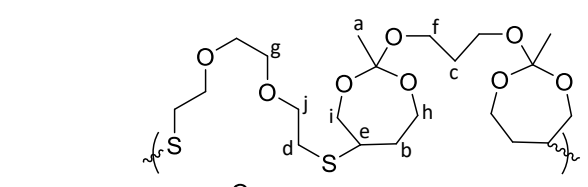

\section{(5c)}

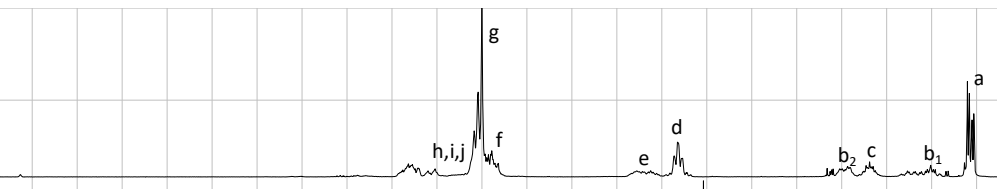

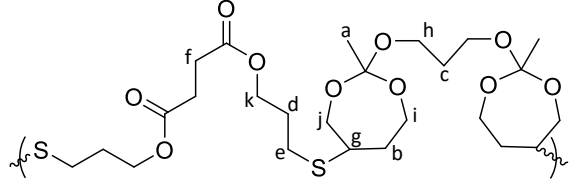

(5d)

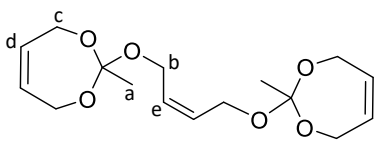

(3c)

e d

ch

\begin{tabular}{lllllllllllllllllllllllllllllllllll}
\hline & 6.0 & 5.8 & 5.6 & 5.4 & 5.2 & 5.0 & 4.8 & 4.6 & 4.4 & 4.2 & 4.0 & 3.8 & $\begin{array}{c}3.6 \\
\delta(\mathrm{pm})\end{array}$ & 3.2 & 3.0 & 2.8 & 2.6 & 2.4 & 2.2 & 2.0 & 1.8 & 1.6 & 1.4 & 1.2 & 1.0
\end{tabular}

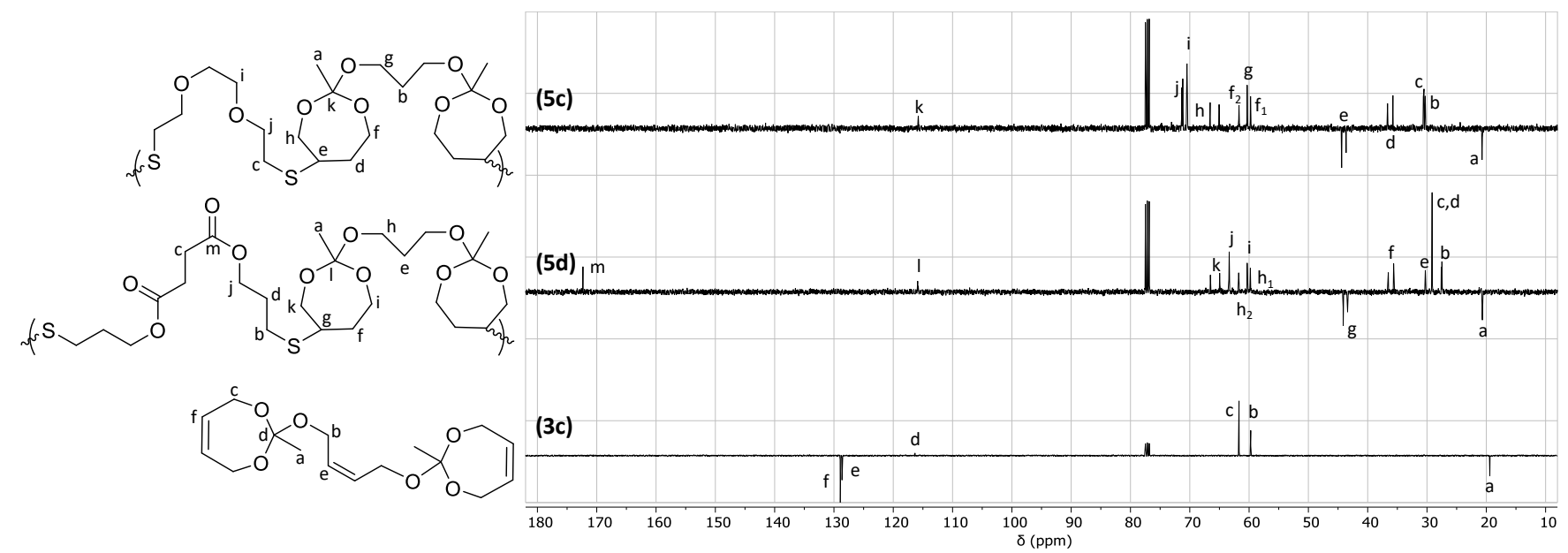

Figure S9. ${ }^{1} \mathrm{H}$ and ${ }^{13} \mathrm{C}$ NMR spectra of remaining polymeric compounds (5c, 5d) and network precursor OE (3c) $\left(\mathrm{CDCl}_{3}, 298 \mathrm{~K}\right)$. Top: ${ }^{1} \mathrm{H}$ NMR

(400 MHz); bottom: ${ }^{13} \mathrm{C}$ NMR $(300 \mathrm{MHz})$.

(1) Plikk, P.; Tyson, T.; Finne-Wistrand, A.; Albertsson, A. C., Mapping the characteristics of the radical ring-opening polymerization of a cyclic ketene acetal towards the creation of a functionalized polyester. J. Polym. Sci. A Polym. Chem. 2009, 47 (18), 4587-4601. doi:10.1002/pola.23511 